\title{
Understanding the Socioeconomic and Geographical Characteristics of Beneficiaries Receiving a Comprehensive Medication Review
}

\author{
Joshua Chou, PharmD, MS, BCGP; Karen Pellegrin, PhD, MBA; Catherine E. Cooke, PharmD, MS, BCPS; \\ Barbara Zarowitz, PharmD, BCPS, BCGP; Alexandra Hanlon, PhD; Alicia Lozano, MS; \\ and Nicole J. Brandt, PharmD, MBA, BCGP
}

\begin{abstract}
BACKGROUND: Medicare Part D sponsors are required to offer medication therapy management (MTM) programs to eligible beneficiaries. Recent studies have demonstrated that there have been racial/ethnic disparities in MTM eligibility criteria. For example, compared with non-Hispanic White beneficiaries, Hispanic and non-Hispanic Black beneficiaries are less likely to be eligible for MTM. However, there is limited evidence for socioeconomic and geographical characteristics of those who are eligible and receive MTM services.
\end{abstract}

OBJECTIVE: To describe the demographic, socioeconomic, and geographic characteristics of Medicare beneficiaries who received MTM services.

METHODS: As part of a previous study, a national survey evaluated a convenience sample of perspectives of Medicare beneficiaries on the MTM standardized format. The survey was distributed through Medicare Part D plans to beneficiaries receiving MTM services from 2017-2018. As part of the survey, respondents could provide their ZIP codes. Geographical variables, such as the National Center for Health Statistics (NCHS) urbanrural classification scheme and economic research service (ERS) county typology codes, were then applied to respondents' ZIP codes, allowing for the classification of counties or census tracts by urbanization and economic dependence measures. Descriptive statistics are reported for demographic, geographical, and socioeconomic information.

RESULTS: Of the $\mathbf{3 0 0}$ (of 434) respondents who provided their ZIP codes, $51.3 \%$ were aged $65-74$ years; $50 \%$ were male; and $66.7 \%$ had at least a college education. There were $82.7 \%$ who self-identified as White, while only $8 \%$ self-identified as Hispanic or Black/African American. The majority of respondents $(58.4 \%)$ lived in large metropolitan areas as defined by the NCHS urban-rural classification scheme. Respondents' counties were characterized by economic dependence with $14.0 \%$ of respondents living in federal/state government-dependent counties and $12.7 \%$ living in recreation-dependent counties.

CONCLUSIONS: The majority of respondents who provided their ZIP codes identified themselves as White and lived in large metropolitan areas. Respondents who identified themselves as Hispanic or Black/African American were not well represented. This study provides geographical and socioeconomic characteristics of Medicare beneficiaries who received MTM services and highlights racial/ethnic differences. Further work is needed to confirm geographical and socioeconomic disparities among beneficiaries who received MTM services.

J Manag Care Spec Pharm. 2020;26(10):1276-81

Copyright $\odot 2020$, Academy of Managed Care Pharmacy. All rights reserved.

\section{What is already known about this subject}

Racial/ethnic inequities have been noted in medication therapy management (MTM) program eligibility.

It is unclear if there are also racial/ethnic disparities in those who receive MTM program services.

\section{What this study adds}

This study describes geographical and socioeconomic characteristics of Medicare beneficiaries who received MTM program services

The majority of beneficiaries within the study population were White and lived in large metropolitan areas, while respondents who were Hispanic or Black/African American were not well represented.

$\mathrm{T}$ he passage of the Medicare Modernization Act (MMA) in 2003 created a mechanism to provide coverage for pharmaceuticals and medication therapy management (MTM) services to Medicare beneficiaries. Under the MMA, each Medicare Part D sponsor is required to offer eligible Medicare beneficiaries an MTM program. ${ }^{1,2}$ Since part of the MTM program requirements stipulated by later regulation (\$423.153[d]), a Part D sponsor must offer an annual comprehensive medication review (CMR) between the beneficiary and a qualified MTM provider where medications are reviewed, drug therapy problems are identified, and a plan for resolution is developed. ${ }^{1,2}$

Beneficiaries are eligible for MTM services if they have multiple chronic diseases, take multiple Part D medications, and are likely to exceed an annual predetermined cost threshold for covered Part D medications. ${ }^{2-4}$ Although the Centers for Medicaid \& Medicare Services strives to increase enrollment for MTM programs, there have been racial/ethnic inequities noted in MTM eligibility. ${ }^{5}$ For instance, using 2004-2005 data from the Medicare Current Beneficiaries Survey, Wang et al. (2014) conducted a retrospective analysis to examine if racial/ethnic disparities in health status, health services utilizations/costs, and medication use patterns differed between MTM-ineligible beneficiaries and MTM-eligible beneficiaries. ${ }^{5}$ These investigators found that there were larger racial/ethnic 


\section{Understanding the Socioeconomic and Geographical Characteristics of Beneficiaries Receiving a Comprehensive Medication Review}

disparities in self-reported good health status among MTM ineligible beneficiaries compared with MTM eligible beneficiaries. Moreover, because the MTM eligibility criteria are based primarily on prescription drug cost and medication use, racial/ ethnic minorities may be disproportionately affected because minorities historically use fewer prescription drugs and spend less money on medications compared with Whites. ${ }^{6}$

To date, it is unclear if the observed racial/ethnic disparities in MTM eligibility are seen in those who actually receive MTM services because socioeconomic and geographical characteristics of those who received MTM services are not readily available. The objective of this study was to describe the demographic and geographic characteristics of a convenience sample of Medicare beneficiaries who received MTM services.

\section{Methods}

A previous study performed by several of the authors of this article assessed the perspectives of Medicare beneficiaries on the utility of the Medication Therapy Management (MTM) Standardized Format (SF). ${ }^{7}$ A link to an electronic survey was distributed nationally by Medicare Part D plans that had partnered with the research team through the AMCP MTM Advisory Group. The plans distributed the survey link electronically to a sample of beneficiaries who received MTM services from 2017-2018. Additional details of the survey design and dissemination can be found in the corresponding publication. ${ }^{\top}$

As part of the survey, respondents could provide demographic information, including age, gender, race/ethnicity, ZIP code, and education. They could also provide other information such as self-health rating and number of medications. Respondents' geographic states were identified from the respondents' ZIP codes and then categorized into geographic regions according to the U.S. Census Bureau. ${ }^{8}$ Codes and classification schemes were then applied to the respondents' ZIP codes, allowing for the classification of counties or census tracts by urbanization and economic dependence measures. These geography-based variables include the National Center for Health Statistics (NCHS) urban-rural classification scheme and economic research service (ERS) county typology codes (see the Supplementary Appendix for a more detailed description of the NCHS and ERS geography-based variables, available in online article).

The NCHS urban-rural classification scheme is a 6-level classification scheme for counties within the United States that allows for the NCHS to study associations between urbanization level and health. ${ }^{9}$ The categories range from the most urban category of large metropolitan areas to the most rural category that includes nonmetropolitan "noncore" counties. Nonmetropolitan "noncore" counties are defined as counties that are not designated as core-based statistical areas, which include metropolitan and micropolitan statistical areas. ${ }^{9}$

\section{TABLE 1 Demographics and Health Status of Survey Respondents $(\mathrm{N}=300)$}

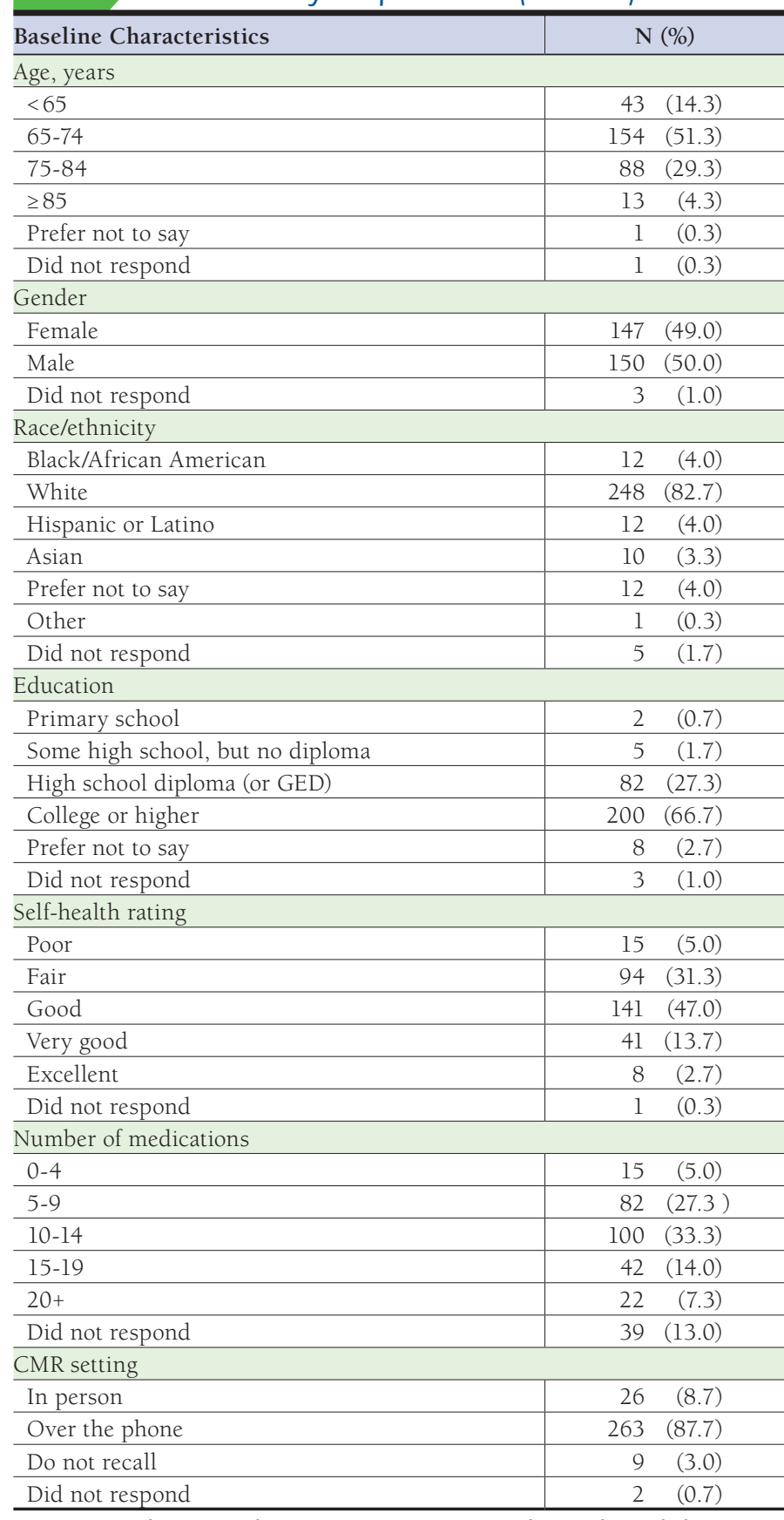

$C M R=$ comprehensive medication review; GED = general equivalency diploma

On the other hand, ERS typology codes classify U.S. counties according to 6 mutually exclusive categories of economic dependence and 6 overlapping categories of policyrelevant themes. ${ }^{10}$ Economic dependence types include mining, manufacturing, federal/state government, recreation, and 


\begin{tabular}{|c|c|}
\hline $\begin{array}{cc}\text { TABLE 2) } & \text { Geo } \\
& \text { Char } \\
& (\mathrm{N}=3\end{array}$ & $\begin{array}{l}\text { Geographical and Socioeconomic } \\
\text { Characteristics of Survey Respondents } \\
(\mathrm{N}=300)\end{array}$ \\
\hline Characteristics & $\mathrm{N}(\%)$ \\
\hline \multicolumn{2}{|l|}{ Geographic region } \\
\hline Northeast & $35(11.7)$ \\
\hline Midwest & $59(19.7)$ \\
\hline South & $92(30.6)$ \\
\hline West & $114(38.0)$ \\
\hline \multicolumn{2}{|c|}{ NCHS urban-rural classification scheme for counties (2013) } \\
\hline Large central metro & $104(34.7)$ \\
\hline Large fringe metro & $71(23.7)$ \\
\hline Medium metro & $68 \quad(22.7)$ \\
\hline Small metro & $27 \quad(9.0)$ \\
\hline Micropolitan & $21 \quad(7.0)$ \\
\hline Non-core & $9 \quad(3.0)$ \\
\hline \multicolumn{2}{|c|}{ Economic dependence county indicator } \\
\hline Nonspecialized & $181 \quad(60.3)$ \\
\hline Mining & $4 \quad(1.3)$ \\
\hline Manufacturing & $35(11.7)$ \\
\hline Federal/state government & $42(14.0)$ \\
\hline Recreation & $38 \quad(12.7)$ \\
\hline \multicolumn{2}{|c|}{ Low employment county indicator } \\
\hline No & $279(93.0)$ \\
\hline Yes & $21 \quad(7.0)$ \\
\hline \multicolumn{2}{|c|}{ Population loss county indicator } \\
\hline No & $266(88.7)$ \\
\hline Yes & $34(11.3)$ \\
\hline \multicolumn{2}{|c|}{ Retirement destination county indicator } \\
\hline No & $248 \quad(82.7)$ \\
\hline Yes & $52(17.3)$ \\
\hline \multicolumn{2}{|c|}{ Classification of counties by level of poverty over 3 decades } \\
\hline All other counties & $295(98.3)$ \\
\hline Persistent poverty county & $5 \quad(1.7)$ \\
\hline
\end{tabular}

nonspecialized counties. The policy-relevant types include low employment, persistent poverty, population loss, and retirement destination.

Descriptive statistics were reported for demographic, geographical, and socioeconomic information. SPSS software, version 26, was used for all statistical analyses (IBM, Armonk, NY). The protocol for this study was reviewed and approved under exempt status by the University of Maryland Baltimore Institutional Review Board.

\section{Results}

There were 434 unique surveys that were received from the previous study. ${ }^{7}$ The survey response was $4.3 \%$. Of the 434 unique respondents, 300 provided their ZIP codes.

\section{Demographic Information}

Among respondents providing their ZIP codes, 51.3\% were aged $65-74$ years; $50 \%$ were male; and $66.7 \%$ had at least a college education. There were $82.7 \%$ who identified themselves as White, while only $8 \%$ self-identified as Hispanic or Black/ African American. A sizeable portion of the respondents rated their health as either fair $(31.3 \%)$ or good $(47 \%)$. The vast majority $(81.9 \%)$ of the respondents were taking at least 5 medications, and $54.6 \%$ were taking at least 10 medications. In addition, most respondents received their CMRs over the phone (87.7\%; Table 1).

\section{Geographical Characteristics}

The majority of respondents (58.4\%) lived in large metropolitan areas (large central metro and large fringe metro) as defined by the NCHS urban-rural classification scheme (Table 2). These metropolitan areas are defined by the NCHS as counties in metropolitan service areas having a population of at least 1 million. ${ }^{9}$ Respondents' counties were also classified by ERS county typology codes based on economic dependence. While most respondents' counties were nonspecialized (60.3\%), 14\% of respondents lived in federal/state government-dependent counties, and $12.7 \%$ lived in recreation-dependent counties. Finally, respondents' counties were also classified by ERS county typology codes by county policy types. Most of the respondents' counties were not determined to be low employment counties (93\%), population loss counties (88.7\%), or persistent poverty counties (98.3\%). However, $17.3 \%$ of respondents' counties were classified as retirement destination counties.

\section{Discussion}

Racial/ethnic disparities remain an ongoing challenge within the U.S. health care system. Individuals of color still face significant disparities in access, coverage, and utilization of care, as well as health status and outcomes. ${ }^{11}$ Among Medicare beneficiaries, compared with White beneficiaries, Black and Hispanic beneficiaries are more likely to have limited financial resources, report poorer health status, and have a more difficult time getting needed care. ${ }^{12}$ What is not as well known, is whether racial/ethnic disparities exist in those who actually receive MTM services, given that MTM is an optional service offered to all Medicare beneficiaries. The findings from this study help to fill this knowledge gap and describe socioeconomic characteristics of Medicare beneficiaries who received MTM services. The study population was fairly homogeneous as most respondents were White, lived in large metropolitan areas, and in counties that were affluent. The study population had a low representation of Hispanic and Black/African American beneficiaries, which is consistent with the racial/ ethnic disparities noted throughout the literature. ${ }^{13-15}$ 
Interestingly, a recent study by Coe et al. (2019) noted that Black older adults were more likely to receive a CMR compared with White, Asian, Native American, Indian, and Hispanic older adults. ${ }^{16}$ The study also showed that a higher proportion of Black and Hispanic older adults were likely to be enrolled in MTM. Unlike our study, the study population for Coe et al. included Medicare beneficiaries who were eligible for, offered, and received CMRs in 2013 and 2014. Our study population comprised Medicare beneficiaries who received CMRs and then responded to an electronic survey regarding the CMR. While this distribution method was efficient, it likely resulted in a lower representation of those populations with decreased access to computers and email. For instance, according to a Pew Research Center report conducted in 2015, 97\% of individuals who live in households with an annual income of $\$ 75,000$ or more report that they are internet users. ${ }^{17}$ On the other hand, $74 \%$ of individuals who live in households with an annual income of $\$ 30,000$ or less report that they are internet users. The Pew Research Center report also estimated that 85\% of White individuals use the internet, compared with $81 \%$ for Hispanic and $78 \%$ for Black, non-Hispanic individuals. As a result, the distribution method could have accounted for the difference in results between the studies.

Studies have demonstrated the benefit of MTM services. Positive health outcomes for those receiving MTM services include a decrease in inpatient visits, lower admission rates, lower rates of emergency department visits, reduction in total health care costs, and higher medication adherence. ${ }^{18-21}$ Moreover, as shown by the IMPACT study, MTM services in underserved communities have resulted in significant improvement in clinical markers associated with diabetes mellitus, such as glycated hemoglobin Alc, low-density lipoprotein, and triglyceride concentrations. ${ }^{22}$ The improvement in clinical outcomes was attributed to a multipronged implementation strategy that included addressing social determinants of health (e.g., providing grocery store gift cards, transportation passes, and free testing supplies); providing individualized diabetes education and therapeutic recommendations; and patientcentered collaboration among the interprofessional health care team. ${ }^{22}$ Thus, such implementation strategies and perhaps others are likely important for MTM services to improve patient outcomes in minority populations.

Given the benefits of MTM, it is therefore concerning that there may be racial/ethnic disparities in beneficiaries receiving MTM services. The MTM eligibility criteria may perpetuate racial/ethnic disparities in access to health care services. ${ }^{5,6,23} \mathrm{In}$ addition, sociocultural differences between patients and health care professionals can result in a lack of understanding between the 2 parties. ${ }^{13}$ If these differences are not fully accepted or embraced, cultural or linguistic barriers arising from the health care system may result in a negative effect on communication and trust, encouraging certain racial/ethnic groups to decline health care services. ${ }^{13}$ As such, because beneficiaries can freely opt-out of MTM programs, beneficiaries encountering cultural or linguistic barriers may decline MTM programs because of dissatisfaction with their health care or distrust with their health care providers. ${ }^{13}$

On the other hand, MTM program enrollment differences, or low enrollment in general, could be due to lack of perceived benefit or need for MTM services by eligible Medicare beneficiaries. ${ }^{24,25}$ For example, when Doucette et al. (2007) surveyed Medicare beneficiaries on their perceptions of pharmacist-provided counseling and MTM services, the beneficiaries had a more favorable attitude toward counseling activities compared with MTM services. ${ }^{24}$ The authors attributed this finding to the novelty of MTM services at the time of the study. However, a decade after the findings of Doucette et al. were published, Medicare beneficiaries are still generally unaware of MTM activities. A recent study done by Taylor et al. (2018) demonstrated that Medicare beneficiaries were generally unaware of MTM program activities, as well as the benefits of MTM programs. ${ }^{26}$

More work remains to confirm if there are racial/ethnic disparities in beneficiaries receiving MTM services, potentially using other data sources such as Medicare Part D claims data. In addition, MTM program CMR completion rate for Medicare Advantage Prescription Drug (MAPD) plans has steadily increased from $30.9 \%$ in 2016, $45.6 \%$ in 2017 , and $77 \%$ in 2019. 27,28 Similarly, although standalone prescription drug plans' (PDPs) CMR completion rate is generally lower than that of MAPDs, CMR completion rates also increased from 15.4\% in 2016, 25.3\% in 2017, and 44\% in 2019..$^{27,28}$ While CMR rates have increased, future MTM program designers need to further understand how to better align MTM programs to the needs of minorities and communities. ${ }^{3}$

\section{Limitations}

This study has some limitations to consider. While this study represents a step toward better understanding the socioeconomic characteristics of Medicare beneficiaries who received MTM services, the study population was a convenience sample. As such, the study population was not representative of Medicare beneficiaries nationwide, and the results were not generalizable. Moreover, the survey distribution method might have reduced participation among groups of beneficiaries, especially those with limited financial resources or belonging to certain racial/ethnic groups. However, the demographic information collected in the survey was reflective of the greater Medicare beneficiary population. ${ }^{7}$ According to the Medicare Payment Advisory Committee report, $74 \%$ of the beneficiaries were White, compared with $82.7 \%$ of our respondents who identified themselves as White. ${ }^{29}$ Additionally, the majority of respondents lived in large metropolitan areas as defined by the NCHS urban-rural classification scheme, which is generally 


\section{Understanding the Socioeconomic and Geographical Characteristics of Beneficiaries Receiving a Comprehensive Medication Review}

similar to the distribution of the U.S. population based on 2013 Centers for Prevention and Disease Control data. ${ }^{30}$

Although selection bias is a concern with the study population, this study took an important step toward identifying possible disparities between beneficiaries receiving MTM services. This study, in combination with future studies that use nationally representative datasets can help better depict who is receiving MTM services but, more importantly, who is not receiving MTM services.

\section{Conclusions}

The majority of respondents who provided their ZIP codes identified themselves as White and lived in large metropolitan areas. Respondents who identified themselves as Hispanic or Black/African American were not well represented. This study provides a convenience sample of geographical and socioeconomic characteristics of Medicare beneficiaries who received MTM services and highlights racial/ethnic differences. Further work is needed to confirm geographical and socioeconomic disparities among beneficiaries who receive MTM services.

\section{Authors}

JOSHUA CHOU, PharmD, MS, BCGP; CATHERINE E. COOKE, PharmD, MS, BCPS; BARBARA ZAROWITZ, PharmD, BCPS, BCGP; and NICOLE J. BRANDT, PharmD, MBA, BCGP, University of Maryland School of Pharmacy, Baltimore. KAREN PELLEGRIN, PhD, MBA, University of Hawai i at Hilo. ALEXANDRA HANLON, $\mathrm{PhD}$, and ALICIA LOZANO, MS, Virginia Tech, Blacksburg, Virginia.

AUTHOR CORRESPONDENCE: Joshua Chou, PharmD, MS, BCGP, Johns Hopkins Hospital, Baltimore, MD 21287.

Tel.: 301.335.0784; Email: jchou21@jhmi.edu.

\section{DISCLOSURES}

No outside funding supported this study. Pellegrin is a member of the AMCP MTM Advisory Group. The other authors have nothing to disclose.

\section{REFERENCES}

1. Brandt NJ. Cooke CE. Centers for Medicare and Medicaid Services support for medication therapy management (enhanced medication therapy management): testing strategies for improving medication use among beneficiaries enrolled in Medicare Part D. Clin Geriatr Med. 2017;33(2):153-64.

2. Prescription drug plan sponsors' access to Medicare Parts A and B claims data extracts. 42 CFR § 423.153. April 16, 2019. Available at: https:// www.govregs.com/regulations/title42_chapterIV_part423_subpartD_ section423.153. Accessed August 18, 2020.

3. Wang J, Shih YT, Qin Y, et al. Trends in Medicare Part D medication therapy management eligibility criteria. Am Health Drug Benefits. 2015;8(5):247-55
4. Centers for Medicaid \& Medicare Services. 2010 Medicare Part D Medication Therapy Management (MTM) Programs. June 8, 2010. Available at: https://www.cms.gov/Medicare/Prescription-Drug-Coverage/ PrescriptionDrugCovContra/downloads/MTMFactSheet_2010_06-2010_ final.pdf. Accessed August 18, 2020.

5. Wang J, Qiao Y, Tina Shih YC, et al. Potential health implications of racial and ethnic disparities in meeting MTM eligibility criteria. Res Social Adm Pharm. 2014;10(1):106-25.

6. Spivey CA, Qiao Y, Wang J, et al. Comparative effectiveness of medication therapy management eligibility criteria across racial/ethnic groups. J Am Geriatr Soc. 2019;67(3):581-87.

7. Brandt NJ, Cooke CE, Sharma K, et al. Findings from a national survey of Medicare beneficiary perspectives on the Medicare Part D Medication Therapy Management Standardized Format. J Manag Care Spec Pharm. 2019;25(3):366-91. Available at: jmcp.org/doi/10.18553/jmcp.2019.25.3.366.

8. United States Census Bureau. Terms and definitions. December 5, 2016. Available at: https://www.census.gov/programs-surveys/popest/guidancegeographies/terms-and-definitions.html. Accessed August 18, 2020.

9. Centers for Disease Control and Prevention. NCHS Urban-Rural Classification Scheme for Counties. June 1, 2017. Available at: https://www. cdc.gov/nchs/data_access/urban_rural.htm. Accessed August 18, 2020.

10. United States Department of Agriculture. Economic Research Service. County typology codes. October 23, 2019. Available at: https://www.ers.usda. gov/data-products/county-typology-codes.aspx. Accessed August 18, 2020.

11. Artiga S, Orgera K. Key facts on health and health care by race and ethnicity. November 12, 2019. Available at: https://www.kff.org/disparitiespolicy/report/key-facts-on-health-and-health-care-by-race-and-ethnicity/. Accessed August 18, 2020.

12. The Henry J. Kaiser Family Foundation. Profile of Medicare beneficiaries by race and ethnicity: a chartpack. March 9, 2016. Available at: https:// www.kff.org/medicare/report/profile-of-medicare-beneficiaries-by-race-andethnicity-a-chartpack/. Accessed August 18, 2020.

13. Betancourt JR, Green AR, Carrillo JE, Ananeh-Firempong O. Defining cultural competence: a practical framework for addressing racial/ethnic disparities in health and health care. Public Health Rep. 2003;118(4):293-302.

14. Egede LE. Race, ethnicity, culture, and disparities in health care. J Gen Intern Med. 2006;21(6):667-69.

15. American College of Physicians. Racial and ethnic disparities in health care, updated 2010. May 13, 2010. Available at: https://www.acponline.org/acp_ policy/policies/racial_ethnic_disparities_2010.pdf. Accessed August 18, 2020.

16. Coe AB, Adeoye-Olatunde OA, Pestka DL, et al. Patterns and predictors of older adult Medicare Part D beneficiaries' receipt of medication therapy management. Res Soc Adm Pharm. 2020;16(9):1208-14.

17. Perrin A, Duggan M. Americans' internet access: 2000-2015. Pew Research Center. June 26, 2015. Available at: https://www.pewresearch.org/ internet/2015/06/26/americans-internet-access-2000-2015/\#internet-usageby-race-ethnicity. Accessed August 18, 2020.

18. Moore JM, Shartle D, Faudskar L, Matlin OS, Brennan TA. Impact of a patient-centered pharmacy program and intervention in a high-risk group. J Manag Care Pharm. 2013;19(3):228-36. Available at: https://www.jmcp.org/ doi/10.18553/jmcp.2013.19.3.228.

19. Touchette DR, Masica AL, Dolor RJ, et al. Safety-focused medication therapy management: a randomized controlled trial. J Am Pharm Assoc. 2012;52(5):603-12.

20. Isetts BJ, Schondelmeyer SW, Artz MB, et al. Clinical and economic outcomes of medication therapy management services: the Minnesota experience. J Am Pharm Assoc (2003). 2008;48(2):203-11. 


\section{Understanding the Socioeconomic and Geographical Characteristics of Beneficiaries Receiving a Comprehensive Medication Review}

21. Wittayanukorn S, Westrick SC, Hansen RA, et al. Evaluation of medication therapy management services for patients with cardiovascular disease in a self-insured employer health plan. J Manag Care Pharm. 2013;19(5):385-395. Available at: https://www.jmcp.org/doi/10.18553/ jmcp.2013.19.5.385.

22. Bluml BM, Watson LL, Skelton JB, Manolakis PG, Brock KA. Improving outcomes for diverse populations disproportionately affected by diabetes: final results of Project IMPACT: Diabetes. J Am Pharm Assoc (2003). 2014;54(5):477-85.

23. Spivey CA, Wang J, Qiao Y, et al. Racial and ethnic disparities in meeting MTM eligibility criteria based on star ratings compared with the Medicare Modernization Act. J Manag Care Spec Pharm. 2018;24(2):97-107. Available at: https://www.jmcp.org/doi/10.18553/jmcp.2018.24.2.97.

24. Doucette WR, Witry MJ, Alkhateeb F, Farris KB, Urmie JM. Attitudes of Medicare beneficiaries toward pharmacist-provided medication therapy management activities as part of the Medicare Part D benefit. J Am Pharm Assoc (2003). 2007;47(6):758-62.

25. Law AV, Okamoto MP, Brock KA. Perceptions of Medicare Part D enrollees about pharmacists and their role as providers of medication therapy management. J Am Pharm Assoc (2003). 2008;48(5):648-53.
26. Taylor AM, Axon DR, Campbell P, et al. What patients know about services to help manage chronic diseases and medications: findings from focus groups on medication therapy management. J Manag Care Spec Pharm. 2018;24(9):904-10. Available at: https://www.jmcp.org/doi/10.18553/ jmcp. 2018.24.9.904.

27. Gray C, Cooke CE, Brandt NJ. Evolution of the Medicare Part D medication therapy management program from inception in 2006 to the present. Am Health Drug Benefits. 2019;12(5):243-51.

28. Centers for Medicare \& Medicaid Services. Medicare 2020 Part C \& D star ratings technical notes. October 1, 2019. Available at: https://www.cms. gov/Medicare/Prescription-Drug-Coverage/PrescriptionDrugCovGenIn/ Downloads/Star-Ratings-Technical-Notes-Oct-10-2019.pdf. Accessed August 18, 2020.

29. Medicare Payment Advisory Commission. A data book: healthcare spending and the Medicare program. June 2018. Available at: http://medpac. gov/docs/default-source/data-book/jun18_databookentirereport_sec. pdf?sfvrsn=. Accessed August 18, 2020.

30. Ingram D, Franco S. 2013 NCHS Urban - Rural Classification Scheme for Counties. National Center for Health Statistics. Vital Heal Stat. 2014;2(166):1-73. 